\title{
Study of Geomagnetic Storms, Interplanetary Magnetic Field and Solar Wind
}

\author{
Chandrasekhar Bhoj and Lalan Prasad \\ Department of Physics, Govt. P.G. College, Berinag- 262531 Pithoragarh Uttarakhand, India \\ Email: lalitchandu0809192@gmail.com
}

\begin{abstract}
The aim of this paper is to investigate the association of the geomagnetic storms with the IMF for solar cycle 24. Result of the present analysis shows that IMF is geoeffective parameter but its impact varies in accordance with different time periods. The correlation coefficient between Dst and IMF found to be -0.6 for solar cycle 24 .
\end{abstract}

Keywords: Geomagnetic storms, Interplanetary Speed for Solar Cycle 24, magnetic field, Solar wind speed.

\section{Introduction}

A geomagnetic storm is a disturbance of the earth's magnetic field (Akasofu, 1963), arises due to the perturbations in the interplanetary magnetic field (IMF). These perturbations are responsible for the sudden commencements in geomagnetic activities. Burton et al., 1975; Tsurutani et al., 1988; observed that perturbations in the IMF play a key role in producing geomagnetic storms (GS) and suggested that the southward oriented IMFs are more geoeffective compared to the northoriented ones. Gonzalez et al., 1994; found that the peak value of Dst is attained when $\mathrm{Bz}$ (southward component of IMF) also attains its peak value.

Later, Echer et al., 2004 also confirmed the earlier findings that the negative $\mathrm{Bz}$ (southward polarity) is strongly correlated with the Dst (disturbance storm time index). Earlier studies showed that IMF and solar wind play huge roles for the production of magnetic storms but now it is well established that product of IMF and wind speed (VB) is more geoeffective as compared to V or B alone (Russell et al., 1973;
Crooker et al., 1977; Tsurutani et al., 1988; Wang et al., 2009 ). Statistical results obtained by Dwivedi et al., 2009 also confirm that VB is more dominant for the study of Dst as compared to $\mathrm{V}$ or $\mathrm{B}$ alone.

\section{Data analysis and method}

A Chree analysis (Chree, 1913) technique by the superposed epoch method has been done for the present study with the occurrence day of geomagnetic storms (criteria Dst $\leq-50 \mathrm{nT}$ ) as zero day. The daily mean values of the Dst index, IMF and solar wind speed are taken from the omniweb data center (http://omniweb.gsfc.nasa.gov/cgi/nx1.cgi)

\section{Results and Discussion}

Fig.1 is focused on revealing the variation of IMF with Dst index for a part of solar cycle 24 (2011-2017). It is clearly seen from Fig.1 that IMF profile looks like an inverted carbon copy of Dst profile. The minimum peak of Dst concurs with a maximum peak of IMF which shows that strongest increment in IMF 
causes the strongest decrement in Dst which

provides strength to the aforesaid statement

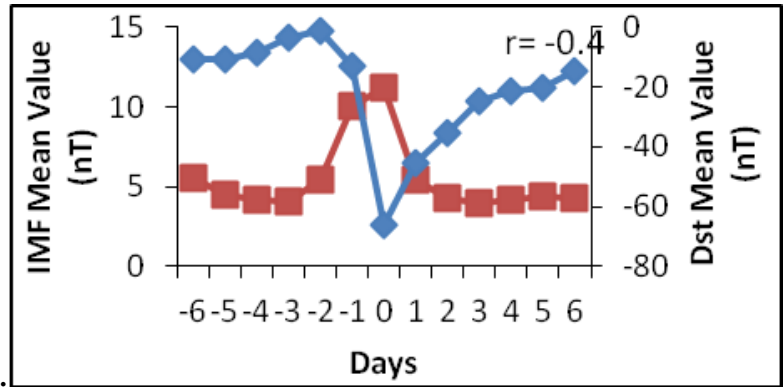

2011

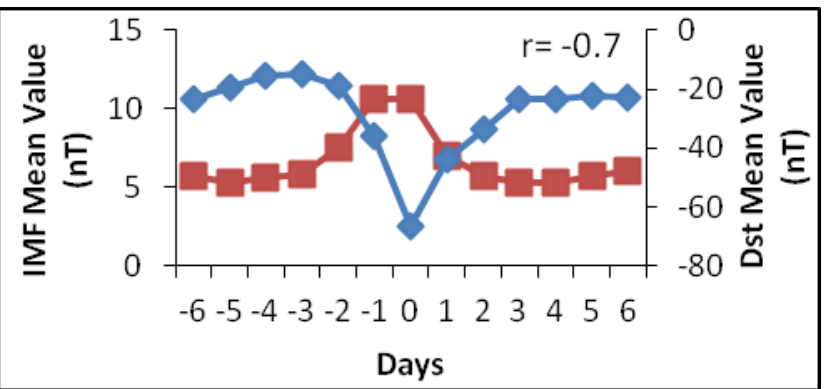

2012

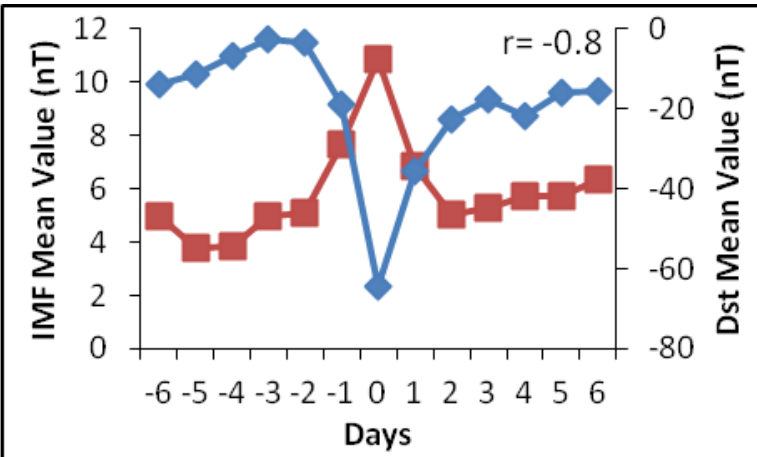

2013

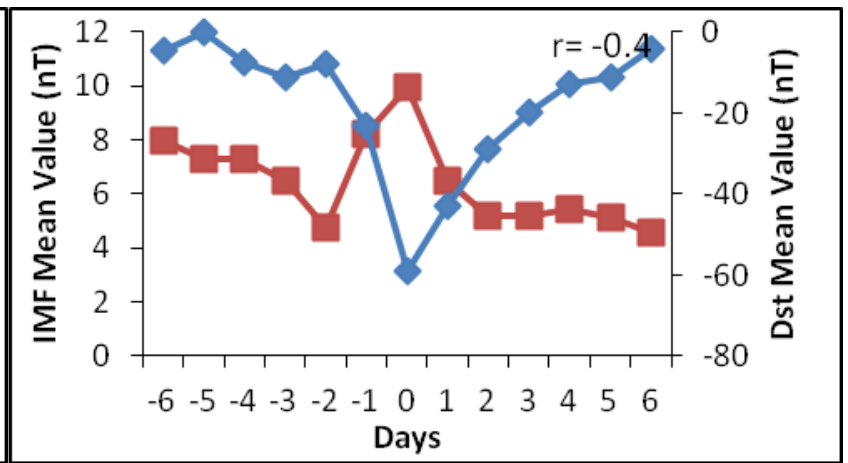

2014

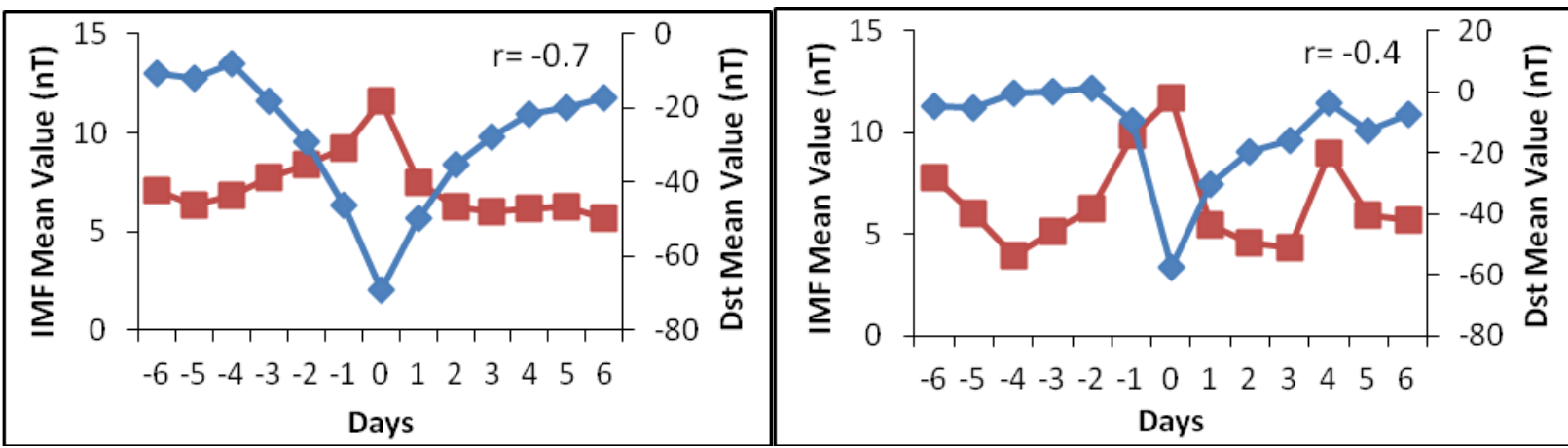

2015

2016

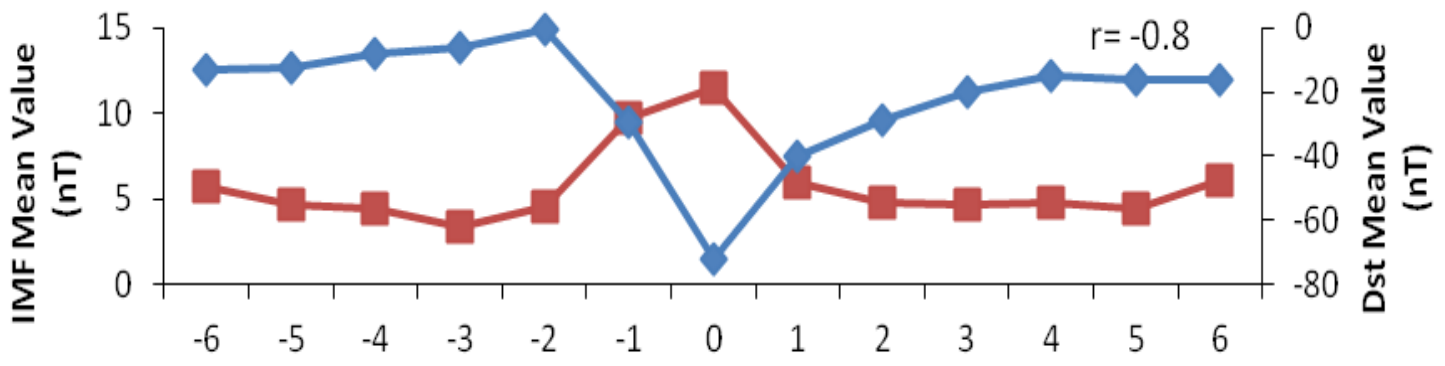

Days

- -imf $\leadsto$ Dst Figure 1 (Color online). The result of Chree analysis from -6 day to +6 days with respect to zero epoch day (Dst $\leq-50 \mathrm{nT}$ )

OSHARAD 28 http://jmr.sharadpauri.org


that IMF is an effective parameter for production of GS (Kharayat et al., 2016). Fig.1 evidently demonstrates the existence of an inverse alliance between IMF and Dst with the average correlation coefficient of -0.6.

\section{Conclusions}

i. The correlation coefficient between Dst and IMF for solar cycle 24 is found to be -0.6.

ii. IMF (B) is geoeffective parameter but its impact varies considerably during different time periods.

\section{Acknowledgements}

The authors are thankful to IUCAA, Pune for content page service and also thankful to ARIES, Nainital for providing library and computing facilities.

\section{References}

Akasofu, S.I., Chapman, S., 1963, Space Sc. Rev. 2, 91Burton, R. K., McPherron, R.L., Russell, C.T., 1975, J. Geophys. Res., 80, 4204- 4214.

Crooker, N. U., Feynman, J., Gosling, J. T., 1977, J. Geophys. Res., 82, 1933-1937.

Chree, C., 1913, Phil. Trans. Roy. Soc., 75, A212.

Dwivedi, V.C., Tiwari, D.P., Agrawal, S.P., 2009, Journal of Geophysical Research, 114, A05108.

Echer, E., Alves, M.V., Gonzalez, W.D., 2004, Solar Phys. 221, 361. Gonzalez, W. D., Joselyn, J.A., Kamide, Y., Kroehl, H.W., Rostoker, G., Tsurutani, B.T., Vasyliunas, V., 1994, J. Geophys. Res., 99, 5771- 5792.
Kharayat H., Prasad L., Mathpal. R., Garia S., Bhatt, B. 2016, Solar Physics, 291 : 603611 94, L11-L15.

Russell, C. T., McPherron, R. L., 1973, J. Geophys. Res., 78, 92-108.Tsurutani, B.T., Gonzalez, W.D., Tang, F., Akasofu, S.I., Smith, E.J., 1988, J. Geophys. Res. 93, 85198531.

Wang, Y. M., Sheeley, N. R., 2009, Astrophys. J., 6

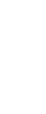

